\title{
A Perspective on NASA Ames Air Traffic Management Research
}

\author{
Jeffery A. Schroeder ${ }^{*}$ \\ NASA Ames Research Center \\ Moffett Field, California 94035
}

\begin{abstract}
This paper describes past and present air-traffic-management research at NASA Ames Research Center. The descriptions emerge from the perspective of a technical manager who supervised the majority of this research for the last four years. Past research contributions built a foundation for calculating accurate flight trajectories to enable efficient airspace management in time. That foundation led to two predominant research activities that continue to this day - one in automatically separating aircraft and the other in optimizing traffic flows. Today's national airspace uses many of the applications resulting from research at Ames. These applications include the nationwide deployment of the Traffic Management Advisor, new procedures enabling continuous descent arrivals, cooperation with industry to permit more direct flights to downstream waypoints, a surface management system in use by two cargo carriers, and software to evaluate how well flights conform to national traffic management initiatives. The paper concludes with suggestions for prioritized research in the upcoming years. These priorities include: enabling more firstlook operational evaluations, improving conflict detection and resolution for climbing or descending aircraft, and focusing additional attention on the underpinning safety critical items such as a reliable datalink.
\end{abstract}

\section{Introduction}

Developing the next-generation-air-transportation system is a prodigious task with many stakeholders. As such, making coherent plans and decisions for its development necessitated the formation of the Joint Planning and Development Office (JPDO). ${ }^{1}$ Even for a constituent organization, such as NASA, developing priorities on where to spend resources is often contentious and not straightforward. Both a consistent set of metrics and a stable approach towards transitional development are lacking. System-wide analysis models are improving; however, their fidelity does not yet allow them to produce unequivocal answers on the priorities. Therefore, when someone rightfully queries, "tell me why you are doing this and not that," discomfort can ensue, as it is difficult to prove that an investment portfolio is sound.

This paper does not provide a systematical analysis to relieve that discomfort; however, it may offer worthwhile insight. That insight comes from a broad reflection on the past and present air-traffic-management research at NASA Ames from the perspective of a single observer. That single observer was a technical manager who supervised the majority of this research at Ames for the last for years, spent nearly a year working for the first JPDO Portfolio Manager, and has 20+ years of experience conducting simulation and dynamics \& controls research.

First, the paper describes past contributions. These past contributions established credibility and expertise that unquestionably led to present research emphases. Ongoing research activities follow descriptions of the past contributions. The paper concludes with suggested new emphases by comparing the present portfolio with future needs. The expressed intent of those suggestions is not to disparage the activities underway but to instead provide constructive feedback for consideration by decisionmakers in the spirit of continuous improvement. With few exceptions, the paper only addresses research conducted in author's organization, which covers approximately $80 \%$ of the total air-traffic-management research at Ames.

\footnotetext{
* Chief, Aviation Systems Division. Presently FAA Chief Scientist for Flight Simulation Systems. Associate Fellow AIAA.
} 


\section{Past Research Contributions}

The contributions in this section predate the author's technical management in the division. However, they are included to give the reader a cohesive background, as these contributions serve as a springboard for the current air-traffic-management research at Ames.

\section{Center TRACON Automation System (CTAS)}

Originally, CTAS was the lynchpin in Ames's research., ${ }^{2,3}$ CTAS was a collection of tools available to aid an air traffic controller or traffic manager. In the collection were the Traffic Management Advisor, the Descent Advisor, and the Final Approach Spacing Tool. The subsequent sections describe these three tools.

Four-dimensional (three in space and one in time) trajectory synthesis is core to all these tools., ${ }^{4,5}$ Trajectories are built based on aircraft flight plans, aircraft performance, winds, radar track data, and expected procedures where flightplans do not exist, such as terminal airspace.

\section{Traffic Management Advisor (TMA)}

The FAA completed its implementation of TMA nationally in Sept. 2007. NASA, MIT Lincoln Laboratory, and the FAA collaboratively developed TMA in the 1990s. ${ }^{6-8}$ TMA allows flow management so that airport demand does not unduly exceed capacity close to the runway.

TMA's use has extended since its first field evaluations, and it now applies in the departure, en route, and arrival flight phases. For departures from airports inside a particular center, TMA schedules those departures to allow them to integrate smoothly into the overhead stream. For en route, TMA can schedule aircraft to arrive at particular points to maximize available capacity. Finally, for arrivals, TMA facilitates the use of all slots between the terminal area boundary and the runway via en route flow management.

As shown in Figure 1, TMA calculates both estimated and scheduled aircraft arrival times to particular points. When an aircraft is between 25-300 miles away, TMA establishes estimated arrival times from the 4dimensional trajectory that it computes using the aircraft's intended route, present radar tracks, winds, separation criteria, terminal procedures, and a model of aircraft performance characteristics. TMA computes an estimated arrival time at each radar update (i.e., every 12 secs).

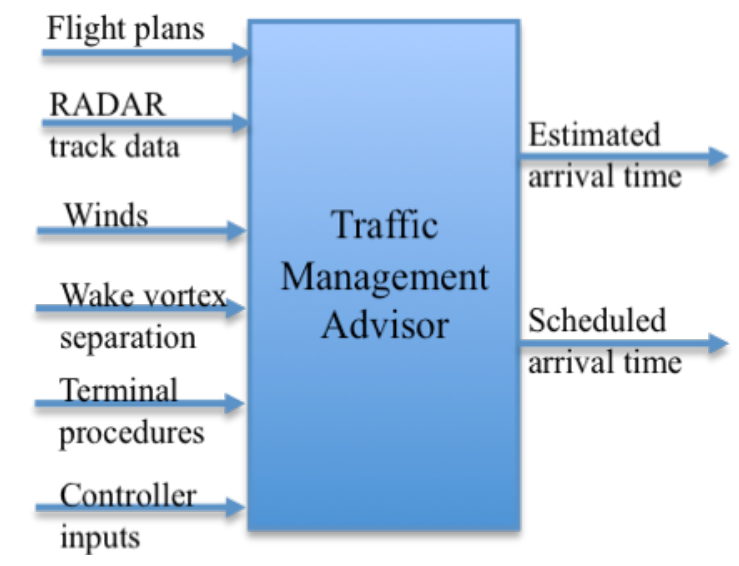

Figure 1 - Inputs and Outputs for the Traffic Management Advisor

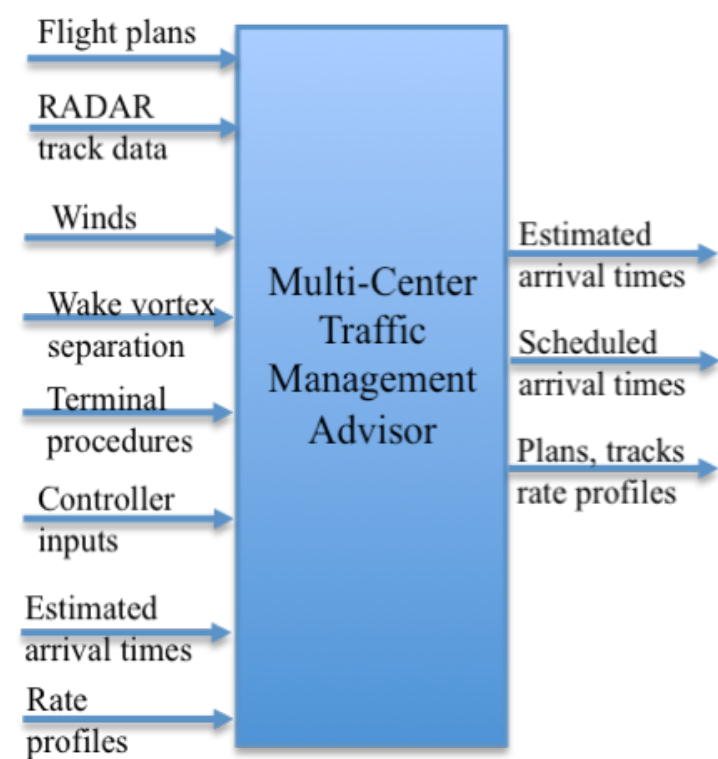

Figure 2 - Inputs and outputs to the Multi-Center Traffic Management Advisor

This estimated arrival time sets the sequence in a modified first-come-first-served order, and these times would be valid if there were no conflicts with other aircraft. If conflicts exist, traffic managers and controllers input constraints, such as acceptance rates or longitudinal separation. TMA then takes these constraints and develops a scheduled arrival time, which will be later than the estimated arrival time. ${ }^{9}$ 
Traffic management coordinators (TMCs) are the main users of TMA. Center TMCs advise terminal-area TMCs on the number of aircraft expected to land in 15-min periods. TMA informs the TMCs visually on when demand will exceed capacity for the decision on when metering is necessary. TMA also provides the metering crossing times and delay advisories to enroute radar controllers. Since TMA shows predicted arrival times of all aircraft to the enroute/terminal-area boundaries and the runway threshold, TMCs can also use this information to improve flow efficiency. Center TMCs can also determine release times for aircraft at airports within the Center's airspace. This provides the precision necessary for departures to enter the arrival flow with minimal workload. The management of flows temporally is particularly valuable when flows merge. TMA provides the scheduled times to the Center sector controllers who are responsible for maintaining the schedule.

During TMA's initial development, a one-month evaluation at Fort Worth Center showed an average 70-sec delay reduction during high-demand periods and a 5\% increase in average airport acceptance rate. The mean error in the meter-fix crossing time reduced by $70-80 \mathrm{secs}$, and the standard deviation dropped by $40 \%$. TMA lowered controller workload and increased job satisfaction according to subjective assessments. ${ }^{8}$ In 2002 , measurements of TMA's benefits at Los Angeles Center showed that time-based metering allowed for $8 \%$ more capacity and a $23 \%$ reduction in delay. ${ }^{10}$

The FAA completed TMA's nationwide deployment in Sept. 2007. Throughput has increased by $3 \%$ or more at airports using TMA. ${ }^{11}$ More consistent flows reduce air and ground holding. Situational awareness has improved, allowing for fewer surprises, early knowledge of spikes in demand, and a common inter-domain picture in real time.

TMA is now available nationwide, and while its use continues to increase, some facilities and controllers are not using it due to a variety of factors such as unfamiliarity and lack of comfort with time-based metering methods. Efforts to reduce these factors are ongoing.

\section{Multi-Center Traffic Management Advisor (McTMA)}

When controllers need to delay flights within their purview to meet constraints, sometimes insufficient airspace is available for the delay maneuvers to meet the schedule and constraints. This is particularly true in dense traffic areas like the northeast corridor of the United States.

NASA, MITRE, and CSC developed McTMA to solve this problem by allowing for distributed scheduling among Centers. This distributed scheduling allows the metering schedules developed by each Center to be coordinated for both efficiency and viability. McTMA looks across contiguous Centers to meet constraints by enabling each Center to absorb enough delay internally so that a downstream Center or sector inherits a manageable problem. The slowing of an aircraft before it reaches its arrival Center to meet its scheduled arrival time enables a broader and more effective span of control over that aircraft.

A field test for Philadelphia-bound flights from four Centers - Boston, New York, Cleveland, and Washington - occurred in 2004. ${ }^{12}$ The evaluations demonstrated reduced airborne delays (averages of 5.9-6.5 mins per flight, down from 7.3-11 mins per flight).

Figure 2 illustrates the inputs and outputs of McTMA. The principal differences between the inputs to McTMA relative to TMA are that the flight plans, radar positions, and controller inputs include not only those within the arrival Center controlling those aircraft but also include that information from the adjacent centers all the way to landing. In addition, the estimated arrival times of aircraft that will cross into that arrival Center's boundary are inputs.

An additional input particularly important to the functioning philosophy of McTMA is the rate profile. The rate profile distributes the restrictions at the runway to the meter fixes and meter points upstream (a meter point is any defined point within a Center). As such, the rate profile allows capacity allocation among resources upstream, which results in an ability to schedule to each point nearly independently. ${ }^{13}$

Due to the priority of implementing TMA nationwide, full McTMA implementation still possibly lies ahead. However, several particularly useful features developed in McTMA, such as metering to a user-defined point (instead of only to the terminal-area boundary and the runway), scheduling departures into overhead streams, and sharing information across Centers will be incorporated into the nationwide deployment of TMA.

\section{Descent Advisor}

Descent Advisor (DA) assists controllers in achieving efficient transition between en route and terminal area flight. ${ }^{14,15}$ While flight management systems have been able to calculate idle descent paths for years, the challenge is to create paths that account for other traffic so that a controller does not have to suspend an efficient descent by requiring a level off. Accurate trajectory prediction is the key enabler, which is a challenge during descents, as altitude, speed, and course are all changing. 
Flight trials of DA took place in 1995 at Denver Center. The trials demonstrated that arrival time errors (mean + standard deviation) of less than 15 secs were achievable.

Figure 3 shows the inputs and outputs used by DA. In addition to the trajectory intent and surveillance data needed for separation, aircraft weight and speed intent are downlinked when available to assist in more accurate trajectory generation in CTAS. TMA provides DA with target scheduled times of arrival that are conflict free. DA then iterates on aircraft speeds, altitude profile, and route to both meet the target scheduled times and maintain separation from other aircraft. The results from DA provide a controller with maneuvers that the aircraft should take to safely meet the scheduled time of arrival.

Building off the results of the DA field trial, improvements continued on its controller interface and on determining effective and uninterrupted conflict-free paths that would allow an aircraft's installed flight management system to plan and execute a vertical profile. Field evaluations in Denver begin in Sept. 2009 to determine the fidelity of the path predictions for situations such as stretching the path to maintain separation.

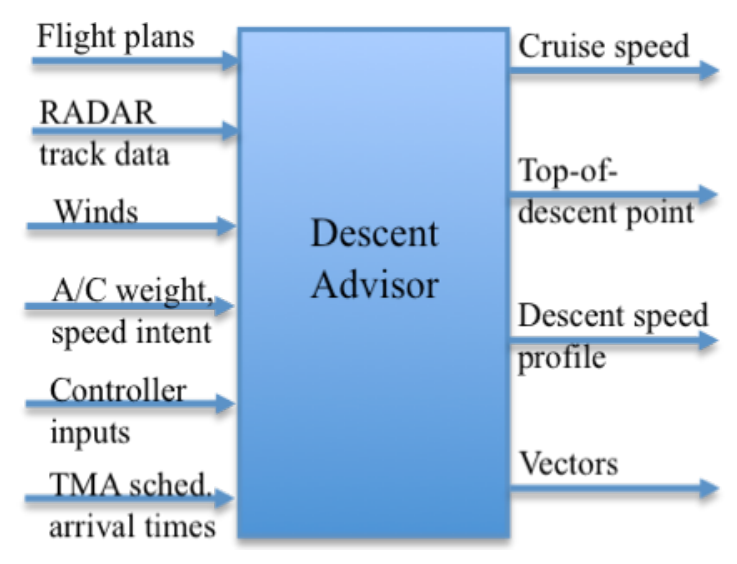

Figure 3 - Inputs and outputs to Descent Advisor

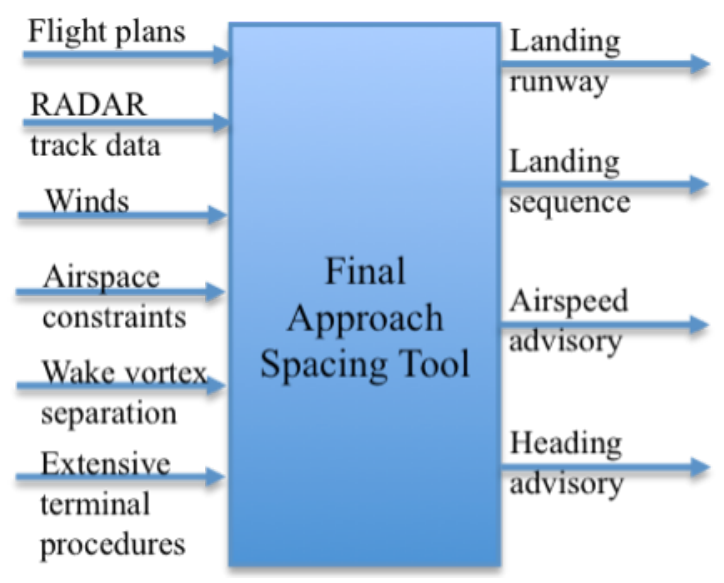

Figure 4 - Inputs and outputs to the Final Approach Spacing Tool

\section{Final Approach Spacing Tool (FAST)}

FAST provided advisories to terminal-area controllers on their radar display for making terminal-area and landing operations more efficient. Figure 4 shows its inputs and outputs. In addition to the inputs required for precise trajectory calculation, FAST needs airspace constraints, wake vortex separation standards, and specific procedures associated with the unique design of a particular airport. FAST then calculates advisories for the landing runway, landing sequence, airspeed and heading. ${ }^{16}$

FAST field tests occurred at Dallas/Fort Worth airport in 1996, but with only the landing runway and landing sequence as outputs. Both the departure and landing rates increased under FAST operation. In addition, the average in-trail separations on approach decreased. With FAST, airport throughput increased over 13\% without negatively impacting controller workload or safety. ${ }^{17}$ While the FAA planned to install FAST at DFW permanently in 1997, that plan changed due to controller concerns that FAST infrequently provided advisories inconsistent with the decisions a controller would make without using FAST.

\section{Direct-To (D2)}

To save flight time, pilots often request permission to cut a corner in their flight plans and proceed directly to a downstream waypoint on their route. ${ }^{18,19}$ The controller tool D2 automatically determines if any aircraft could skip one or more waypoints and proceed directly to such a downstream waypoint. If cutting a corner is possible and the direct route does not substantially deviate from the nominal flight route, the tool advises the controller of the opportunity and the associated timesavings.

Figure 5 shows D2's inputs and outputs. D2 checks the four-dimensional trajectories generated by CTAS to see if a savings of more than one flying minute is possible if an aircraft can proceed to a downstream waypoint without conflicts. Incorporating wind in the trajectory analysis ensures that a direct route saves flying time, not just distance. In addition, D2 displays all conflicts within a controller's airspace over the next 20 mins. 


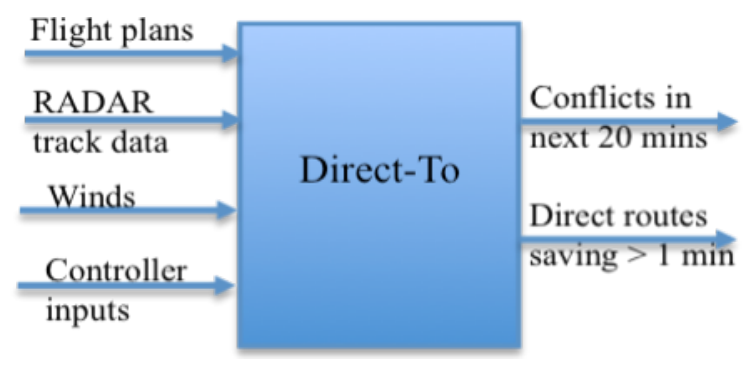

Figure 5 - Inputs and outputs to Direct-To

Operational tests of D2 took place at Fort Worth Center in 2001. The test data suggested that operational implementation in only Fort Worth Center airspace would save 900 flying minutes per day. General controller enthusiasm existed for daily use of D2; however, questions were raised on whether or not D2 might indicate a path was clear of conflict but in disagreement with information from the User Request Evaluation Tool. Due to a lack of funding, the FAA cancelled plans to assess such possible dilemmas.

Subsequent to the operational tests, an interest in D2 developed commercially. In 2008, a joint effort between an industrial partner and NASA adapted D2 to function at airline operation centers. In this environment, when situations arise that suggest an aircraft can skip a waypoint and proceed directly to a downstream waypoint, a suggestion is made to the crew to make that request to air traffic control, thereby saving time and money. Operational tests with the first prototype for only one airline's operations in Fort Worth Center showed considerable promise. Refinements of the prototype are underway for expected tests with another airline.

\section{Surface Movement Advisor (SMA)}

SMA development was a collaborative effort between the FAA and NASA Ames beginning in 1994 with tests occurring at Atlanta-Hartsfield International Airport in $1996 .{ }^{20}$ Presently, it is in use at 16 airports and 11 terminal areas nationwide. SMA falls under the realm of collaborative decisionmaking, as its data provide situational awareness to the airline ramp towers, airline operations centers, and controllers.

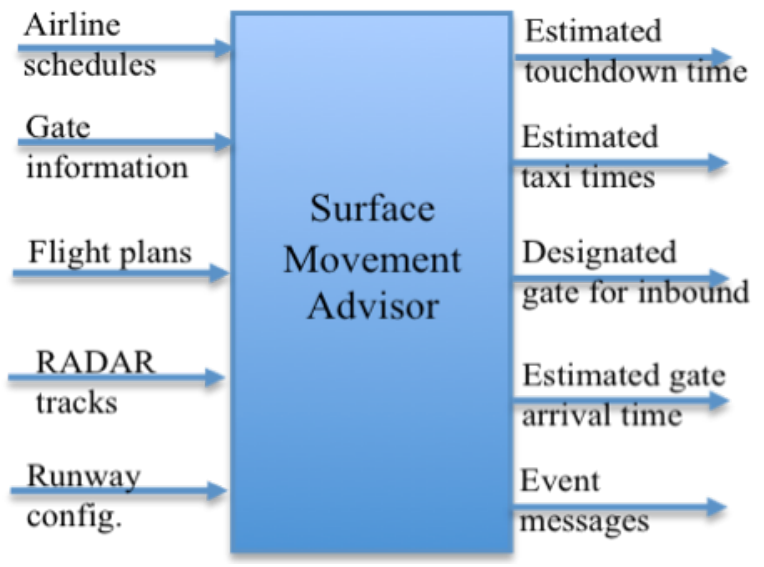

Figure 6 - Inputs and outputs for the Surface Movement Advisor

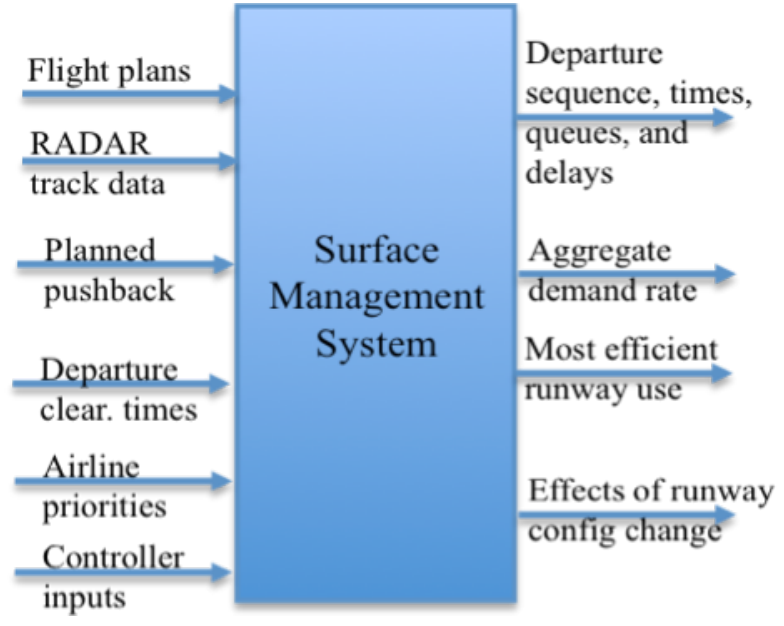

Figure 7 - Inputs and outputs for the Surface Management System

Figure 6 shows the inputs and outputs. SMA combines schedules from the Official Airline Guide, gate information from the Flight Information Display System, flight plans, radar tracks, and the airport's runway configuration to estimate times for landing, taxiing in and out, and arrival at the gate. Various messaging for certain events, such as handoffs between the Center and TRACON, crossing the outer marker, actual touchdown, 
etc. are shared among users to improve decisionmaking and improve the use of airline resources.

It is the author's understanding that other than SMA's use at Atlanta, it is primarily a surveillance data feed for the terminal area. However, much of the functionality and desires expressed by controller's use of SMA led to the development of the Surface Management System, as described next.

\section{Surface Management System (SMS)}

SMS predicts aircraft movements on the surface and terminal area, assesses what would happen for hypothetical traffic management initiatives, and shares this information with appropriate users. ${ }^{21}$ Figure 7 depicts the inputs and outputs to SMS. In addition to flight plans and surface surveillance, the airlines provide their planned pushback times and priorities. The Enhanced Traffic Management System provides the Expected Departure Clearance Times. Controllers can input various "what-if" scenarios and then let SMS predict the results. $^{22}$

Operational tests of SMS took place at Memphis airport in 2003. The results showed that, when looking ahead 30 mins, $75 \%$ of the off times could be predicted with errors less than 15 mins, and $25 \%$ could be predicted with errors less than 4 mins. Aircraft not turning on their transponders when leaving the gate led to significant errors due to the lack of surface surveillance from those aircraft. Both the United Parcel Service and Federal Express use subsequent versions of SMS at the Louisville and Memphis airports respectively.

\section{Future ATM Concepts Evaluation Tool (FACET)}

FACET is a flexible software package used principally for the development and evaluation of traffic-flowmanagement concepts and functions (Bilimoria, et. al, 2001; Sridhar, et. al., 2005). ${ }^{23,24}$ The FAA used FACET in human-in-the-loop simulations to refine the Airspace Flow Program concept. Today, the FAA's reroute conformance monitoring function employs FACET to evaluate how well aircraft are following national traffic management initiatives. The company Flight Explorer has a nonexclusive license to market FACET's capabilities to its subscribers. FACET also figures prominently in the America by Air exhibit at the Smithsonian Institution's National Air and Space Museum.

Figure 8 illustrates the primary inputs and outputs of FACET. Users can input the flight plans, schedules, and track data from a data source of their choice such as the Enhanced Traffic Management System or the Aircraft Situation Display to Industry (ASDI). Many weather input options are available for winds and convective weather. The primary output of FACET is a set of simulated or real 4D trajectories, which are means to an end. Users employ these trajectories to then perform optimization, aid in visualization, or accomplish traffic-flowmanagement functions such as ensuring that a sector's demand does not overly exceed its capacity.

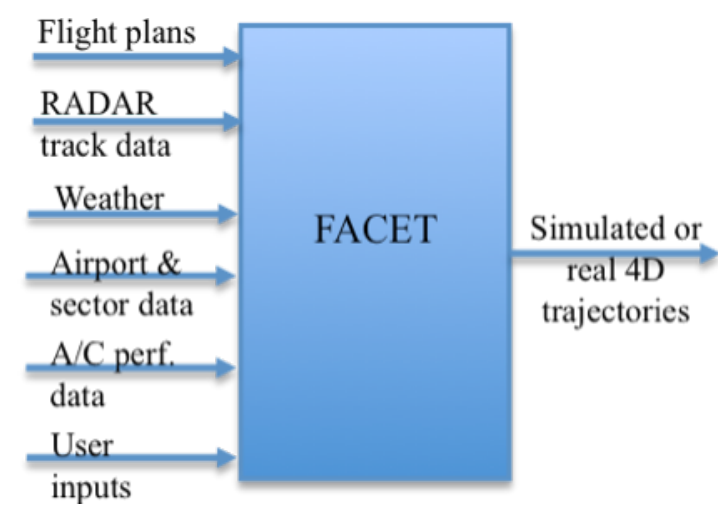

Figure 8 - FACET Inputs and Outputs

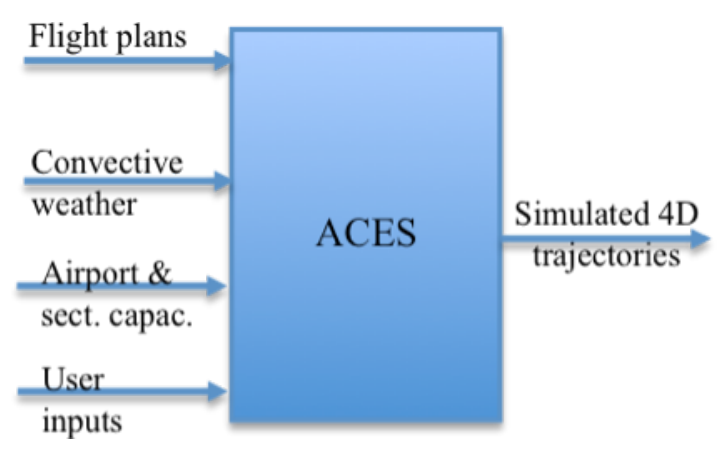

Figure 9 - ACES Inputs and Outputs

\section{Airspace Concept Evaluation System (ACES)}

ACES is a fast-time simulation of the national airspace system that covers operations from gate-to-gate. It is an agent-based architecture with the intent of modeling the first-order effects of future concepts. Its emphasized 
use is more on answering questions of breadth rather than depth; $;{ }^{25-27}$ however, ACES enabled the detailed development of separation assurance algorithms. ${ }^{28}$

Figure 9 illustrates ACES inputs and outputs. Flight plans are available from ASDI to generate initial conditions for nationwide simulations of the air transportation system. Sector capacities are set from the static Monitor Alert Parameters, while airport capacities come from the Aviation System Performance Metrics data. ACES does not commonly model weather explicitly, but instead models it implicitly by adjusting the available capacity of particular resources. Yet, rerouting around weather inputs from MIT Lincoln Laboratory's Convective Weather Avoidance Model was the focus of a recent study. ${ }^{29}$ ACES has users outside of NASA, most notably the Joint Planning and Development Office.

\section{Present Research Contributions}

\section{Separation Assurance}

The vast majority of the separation assurance research at Ames focuses on assisting or automating selected controller functions. The Advanced Automation Concept ${ }^{30}$ is the preferred foundation to achieve that focus. The concept has two principal pieces, with one serving as the safety backup for the other.

The first piece, which is strategic, proposes efficient trajectory changes to keep airplanes safely separated. These changes are efficient in that they consist of both an adjustment to keep an aircraft a safe distance from all other aircraft and a return to the planned route, desired climb, or descent profile. The time span for detecting conflicts is three to twenty minutes into the future. Attempting to predict conflicts beyond 20 mins in the future is pointless, as the uncertainties in the state-of-the-art in trajectory prediction overwhelm the separation standards. The conflict resolutions mimic a controller's duties in ways so as not to request uncommon trajectory changes from a pilot's perspective.

The algorithms embodied in the first piece are complex, and there is no expectation of certifying that software to be safety critical. Here is where the second principal piece of the concept enters. This second piece, which is tactical, is simpler and will be safety critical. It computes trajectory modifications that resolve the conflict from zero to three minutes into the future, and its design solves problems that the first piece does not solve. It is not the intent for the trajectory modifications from this second piece to be efficient. That is, they do not return the aircraft back to its original planned route. They simply keep the airplanes safely apart.

Software prototypes of both of these pieces have been developed and tested for the past several years using real aircraft trajectories from the national airspace system as the starting point. ${ }^{31}$ Simulations of the prototype examined New York, Cleveland, and Fort Worth Centers with the demand increased to three times today's level. ${ }^{32}$ In these simulations, the prototype detected and resolved $100 \%$ of the conflicts. However, these particular results are from simulations without today's typical uncertainties in trajectory predictions.

Thorough human-in-the-loop simulations have examined the strategic first piece described above, and the simulation results have refined the resolution strategies. ${ }^{33}$ The prototype generated acceptable and efficient trajectory changes and appeared feasible for accommodating the anticipated demand growth in the next 20 years.

When simulations include typical uncertainties, the strategic piece of the concept detects and resolves $90 \%$ of the conflicts. ${ }^{34}$ The missed $10 \%$ of conflicts are due to unsatisfactory trajectory prediction for climbing and descending aircraft. This is because, today, airplanes do not fly precision profiles in the vertical plane like the ever-increasing practice in the horizontal plane via required navigation performance.

Several alternatives exist to mitigate these uncertainties. One alternative is to exchange more information between the cockpit and the ground automation, such as weight and speed intent, to improve the trajectory predictions. Another alternative is to expand the conflict region that the algorithm checks. This approach will likely lead to increased false alerts. A third alternative is to use procedural logic similar to what controllers employ today to accommodate the vertical uncertainties.

Figure 10 illustrates the measured $\pm 2 \sigma$ errors in the position prediction 5 mins ahead in the horizontal and vertical planes. These unsatisfactory predictions result in the detection of impending conflicts in less than three minutes, which researchers have somewhat arbitrarily deemed inadequate for a strategic separation assurance function. Efforts are underway to improve these predictions to the likely requirements shown in Figure 10. This may require aircraft to share intent information, such as aircraft weight and climb profile, with air traffic control. 


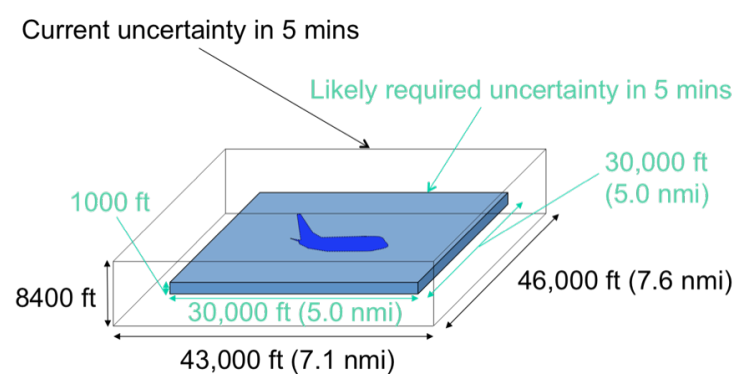

Figure 10 - Trajectory prediction uncertainty

Whether or not the safety-critical backup algorithm detects the $10 \%$ conflicts missed by the first strategic piece under real-world uncertainties has not yet been determined and is underway. This safety-critical algorithm checks for possible conflicts along two trajectories. The first is a constant-velocity, dead-reckoning trajectory similar to that used today by the FAA in their operational conflict alert function. The second trajectory uses the current flight plan.

Researchers tested a prototype of this tactical detection scheme using actual scenarios from 100 operational errors. Compared to today's legacy alerting function, the new prototype detected the conflict for approximately one-half of the cases with at least $1 \mathrm{~min}$ to go before the loss of separation. In addition, the prototype produced $40 \%$ fewer false alerts than the operational conflict alert function. ${ }^{35}$ These promising results have led to FAA consideration for implementing an adaptation of the prototype in a future version of the En Route Automation Modernization System.

A detailed human-in-the-loop simulation also evaluated this tactical detection scheme. ${ }^{36}$ Its addition increased the number of conflicts detected and resolved. In twice today's demand, the scheme detected and resolved $99.9 \%$ of the conflicts. In three times today's demand, it detected and resolved $98.1 \%$ of the conflicts. Late conflict detections, that is, ones detected close to loss-of-separation, are the inimical events that need further attention. This simulation had some uncertainties, as the predicted trajectories did not match the trajectories flown by the simulation exactly; however, researchers have not yet precisely quantified these uncertainties. A key conclusion is that trajectory prediction will need improvement to eliminate late conflict detections that are at risk of going unresolved.

This separation assurance concept is an enabler for routine continuous descent approaches in congested airspace. While flight management systems today can determine paths that allow near-idle approaches from the top-of-descent, controllers often have to issue fuel-consuming and noise-and-emissions producing level offs if traffic conflicts arise. This will only exacerbate as demand increases.

In 2007, a field trial at San Francisco Airport examined continuous descent approaches with early-morning trans-pacific flights. ${ }^{37}$ Four-dimensional trajectories with artificially imposed metering constraints were uplinked to the aircraft, along with recent wind data. The resulting performance showed average time errors at the meter fix of 3 secs for a 25-min prediction horizon. The resulting fuel savings is between 200-3,000 lbs per flight depending on the traffic conditions for heavy aircraft (i.e., B777 and B747).

These tests led to a revision of operational procedures at San Francisco International Airport to allow openloop continuous descent arrivals by several airlines. The overall concept is being refined for future evaluations in more dense traffic at Denver airport during 2011.

\section{Traffic flow management}

The goal of traffic flow management is to effectively schedule the timing and movement of aircraft through the entire airspace system so that the demand does not exceed capacity. Otherwise, delays occur from the supply/demand imbalance.

There are multiple challenges in achieving that goal. First, it is a challenge to predict what the demand is going to be two hours from now. Counting how many predicted aircraft trajectories will enter an airspace region provides satisfactory demand estimates out to only 20 mins owing to trajectory and weather uncertainties. Recently, aggregate models predicted demand out to two hours in advance with standard deviation errors of approximately two aircraft per sector. ${ }^{38}$

A second major challenge is properly accounting for the weather. During the convective weather season, traffic flow managers are especially busy in rerouting aircraft around hazardous weather. Humans accomplish this task based on their experience and ad hoc rules-of-thumb in interpreting displayed weather data. An automation 
challenge is confidently translating weather forecasts into regions that aircraft should avoid. Validation of pilot deviation models and lowering the allowed capacity in regions with forecasted poor weather is ongoing. ${ }^{39}$

Predictions of both the demand and the weather will always be imperfect, so an integrated traffic-flowmanagement structure needs to accommodate these uncertainties and then apply the controls (i.e., ground delay, rerouting, and airborne holding) appropriately. Sequential feedback loops corresponding to strategic (2-8 hr planning horizon) and tactical (0.5-2 $\mathrm{hr}$ planning horizon) timeframes, can intelligently optimize the above full range of controls. ${ }^{40}$

A reasonable question to ask is what are the potential benefits of traffic-flow-management optimization. Today, an approach similar to first-come-first-served assigns ground delays when demand exceeds capacity in a particular airspace region or at an airport; however, strategic optimization can alternatively choreograph the departures of all aircraft arriving into a particular airport to minimize the total delay. It appears possible that delay reductions of over $50 \%$ are possible using in a real-world example of the eastern flow into Chicago. ${ }^{41}$ Optimization also shows promise with the addition of probabilistic information. Specifically, a $7 \%$ delay reduction may be possible by assigning ground delays based on an optimization that included forecasts on when the fog might lift at San Francisco International Airport. ${ }^{42}$ Therefore, these potential benefits justify the value of applying optimization.

After quantifying the demands and weather and then establishing the optimization structure, selection of the appropriate optimization method then follows. Rigorously optimizing all nationwide flows at once has proven to be intractable on existing computers if one wants to optimize flows every 15 mins over, say, 3 hours in the future. A potential way to alleviate that intractability includes aggregating flows into networked regions. ${ }^{43,44}$ This allows a reduction in the number of states by one or more orders of magnitude. The remaining challenge to that approach is determining how to disaggregate the solutions into individual aircraft instructions.

Jointly optimizing the nationwide traffic flow is another possibility by breaking the problem into parallel subproblems. Studies have shown that determining new traffic management controls every 15 mins for all flights over a 3 -hr prediction time using this parallel optimization reduces the computation time to an acceptable level. ${ }^{45}$

The decision remains on whether it is best to use an aircraft-by-aircraft optimization approach or an aggregate/disaggregate approach in the optimization structure described previously. One possibility may be to use both, especially after introducing the additional number of variables required to account for uncertainties. In cases where uncertainty is the greatest, that is, for strategic time horizons of 2 hours or greater, optimization based on aggregate approaches may be feasible, whereas when the uncertainty lessens, the aircraft-by-aircraft optimization approach may be fruitful.

Finally, the collaboration among the individuals or organizations making traffic flow decisions, namely pilots, dispatchers, and traffic-flow managers needs consideration. While NASA is in no position to dictate the levels of authority these parties may make, the optimization tools and processes need to be able to consider them. A framework, based on observations from the field, considers such collaboration. ${ }^{46}$

\section{High-density terminal operations}

Much of the present focus in this research area is on making arrival operations safer and more efficient. Figure 11 shows the concept space with increasing levels of technology required along each axis. Today's stateof-the-art for level-of-automation is vectoring, the level-of-delegation has been increasing with more required navigational performance operations, and the scope-of-optimization is at the individual aircraft level.

At Ames, three areas receive the most attention: 1) increasing capacity with improved time-based scheduling for aircraft capable of advanced procedures, 2) improved safety by automating conflict detection and resolution in the terminal area, and 3 ) increasing capacity using multiple closely-spaced parallel runways. Descriptions follow for each of these three areas.

Improved scheduling aims at extracting more performance from the Traffic Management Advisor by using it in concert with tomorrow's technologies such as area navigation (RNAV), merging and self-spacing that has been delegated to the cockpit, and optimized profile descents. TMA's original design focused on human-intensive operations and navigation performance less than that possible today. With the addition of automation and more accurate and precise navigation, improved scheduling with TMA is possible. Presently, sensitivity analyses are underway that include variations in the time delay distributions in the terminal area at multiple points, time advance, and the time buffers between aircraft.

Automated conflict detection and resolution in the terminal area will provide enhanced safety as the demand and capacity increases in the terminal area. The FAA wants to reduce the number of false alarms generated by the present TRACON Conflict Alert algorithm. Recent simulation results of tool derived from the tactical conflict 
detection and resolution function described earlier shows promise towards reducing those false alarms while increasing detection performance.

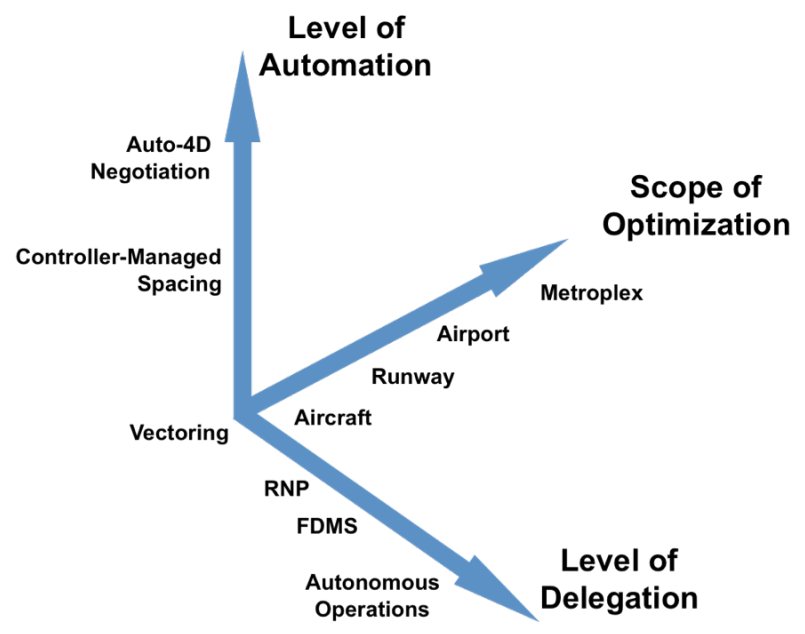

Figure 11 - Concept space for high-density terminal operations ${ }^{47}$

The capacity gains from efficient improvements alone at the nation's busiest airports is not likely to be more than $10 \%$ to $20 \%$. Further gains will be possible either by using more airports or by building more runways and gates at existing airports. One option of adding significant capacity while minimizing some of the environmental impact is to lay a runway down between existing parallel runways. This may allow triple or quadruple closelyspaced parallel approaches if reliable technology and procedures permit such operations. Safety considerations will drive the designs, thus simulations recently examined off-nominal cases for triple approaches with each of the three runways being $750-\mathrm{ft}$ apart. ${ }^{48}$ The results revealed that the workload for closely-spaced arrival operations increases for the crew; however, the workload remains manageable assuming advancements in both flight deck displays and controls.

\section{Dynamic airspace configuration}

This research area has been examining new ways of configuring airspace to maintain a manageable level of workload for controllers as demand inevitably increases. In addition, because demand fluctuates throughout the year, the week, and the day, it may be useful to change airspace geometries safely in concert with those demand changes.

The introduction of airspace complexity may allow the determination of what situations are easier, or more difficult, for a controller to cope with. Simulation results correlated variations in traffic structure with controller workload measurements. ${ }^{49}$ These measurements of complexity are more multifaceted than simply setting a limit on the number of aircraft a controller can manage. Using the complexity measure, and perhaps controlling flow to that measure, may offer a meaningful connection between flow management and automated separation assurance. That is, certain traffic structures may arise that pose difficulty for the automated separation assurance algorithms. If traffic flow management can prevent those situations, naturally the safety case improves.

Several different algorithms including clustering, Voronoi genetic, and mixed-integer programming, have optimized airspace sector geometries to meet various objectives. An even comparison of these approaches showed that the Voronoi genetic algorithm best increases system efficiency with reasonable rises in the number of sectors over today. ${ }^{50}$

Drawing completely new sectors may be impractical or unnecessary at certain times. Instead, recent methods have made minor changes to existing sector geometries to adapt to fluctuating demand. The simplest method is to combine under-utilized sectors to use the controller workforce more efficiently. Currently, the FAA combines sectors at night; however, a systematic approach examined how far the benefits may extend. ${ }^{51}$ The results showed a potential reduction between $40-50 \%$ in the total number of sector-hours (i.e., the number of sectors times the number of hours it is managed) might be possible using a greedy heuristic algorithm. This was under the assumption that the area of specialization among controllers was not a constraint in developing the combinations. The benefits roughly halve if that were a constraint. 
Another area of exploration is generic airspace. Today, sectors often have unique designs, nomenclature, and procedures to accommodate navigation, flow, and safety requirements. This necessitates specialized training, and efforts are in progress to determine whether generic airspace principles can relieve such specialized training and allow more interchangeability between controllers. A recent simulation evaluated a controller information tool that provided useful data to enable a controller to manage an unfamiliar sector. Published results are forthcoming, which showed that the tool aided controllers when managing an unfamiliar sector.

\section{Airport surface}

Intuitively, it is reasonable to assume that tightly arranged surface movements would reduce average taxi times. Whether or not such tight control is possible, it is helpful to know what the maximum benefit might be under perfect conditions.

A constrained taxi optimization simulation answered that question for the Dallas-Ft. Worth airport layout with 25 aircraft released from the east side of the field. ${ }^{52}$ The period for these 25 releases varied from 0 to 30 mins, and the study compared the optimized solution against the first-come-first-served solution. Each scheme enforced safety, runway occupancy, origin and destination timing, and speed constraints. Regardless of the varying release period, the optimization saved an average of approximately six minutes of taxi time per aircraft, which roughly cut the taxi time in half for the 15 min release period.

Optimizing scheduling for departures also offers promise for maximizing throughput and minimizing delay. ${ }^{53,54}$ The simulation results showed that 12 mins of total delay for a 40 -aircraft problem in a Dallas-Fort Worth Airport environment are possible in computations taking less than a tenth of a second.

A real-time simulation investigated controller acceptability of precision-taxi operations under 1.5 times today's traffic level on the east side of Dallas-Fort Worth airport. ${ }^{55}$ The study showed that modifications of the roles and jurisdictions among the local and ground controllers could accommodate precision-taxi operations (this real-time study preceded the development of the constrained optimization solutions described earlier, so the nature of the trajectories was different).

\section{Trajectory Predictions}

An earlier section discussed the errors in predicting an airplane's location five minutes from now. That is but one example of trajectory prediction in air traffic management, as the requirements for trajectory prediction vary with the application. For instance, the trajectory predictions for surface movements, or for traffic flow management movements, are different than for separation assurance.

Several complications arise when discussing trajectory prediction requirements and capabilities. The first is the language used. Precision in language offers collaborative advantages when one discusses the assumptions and modeling in how and by what manner an aircraft flies to a new heading. The lack of language precision has led to unnecessary confusion, which has prevented engineers from leveraging the work of others. Duplication of effort, and increased difficulty for air and ground automation systems to use synchronized trajectories, has been the unfortunate result.

Other complications, in current and past practices, are the hidden initial assumptions, particularly the critical ones that affect performance. Therefore, the subsequent building of the trajectory engines frequently diverges from their inception. The underpinning dynamics used also can create variance. For instance, some trajectory predictions do not use Newton's Laws, but instead use kinematical representations exclusively. General approaches to developing the requirements, comparing the effectiveness, and communicating the content of trajectories are available. ${ }^{56}$

\section{System Level Design and Analysis}

An apt question is how will the advances in the previous individual technologies sum to form a composite benefit, and what is that composite benefit. Accurate answers remain elusive as of yet. A reliable system-level simulation takes years to build, and the approach at present is twofold.

First, in a bottoms-up approach, simulations are assembling two component technologies consisting of an appropriate level of detail to develop confidence in the answers for connecting those two technologies. Examples include connecting strategic weather strategic rerouting with the tactical weather rerouting, which must include separation assurance to prevent reroutes that cause conflicts. When confident results are possible from integrating two components, then three components will be integrated.

The second approach is to develop early answers on what the total system benefits are and then fix the problems that arose during early integration. These estimates presently have large error bars, as the individual technologies and their modeling have not reached a sufficient level of maturity to integrate them dependably. 


\section{Suggestions for Future Research Contributions}

Based upon the past successes and present research contributions, this section offers suggestions on what to do more of and what to do less of in air-traffic-management research. Deciding what to include and its priority were based on the author's opinion gleaned from personally reviewing and approving over 200 publications and presentations from the organization during his tenure, from conducting weekly research meetings with the research principals, and from reviewing and approving accomplishments in annual performance plans for the research staff. Knowledge gained from that experience lends to an individual perspective whose value only the reader can decide.

When an item below is listed under "more of," it was the author's belief that the 2025 Next Generation Air Transportation System (NextGen) objectives of accommodating three times today's capacity while not increasing the accident rate was at risk unless more of that item was addressed. When an item below is listed under "less of," the author believes that meeting the 2025 NextGen objectives do not need the level of effort expended at present on that item. Meeting the 2025 NextGen 3x-capacity objectives outlined in Ref. 1 are aggressive targets, as the recent forecast of the 2025 demand $^{57}$ is approximately half the goal set by the JPDO in 2004.

Items included on the list do not necessarily fall within the purview of NASA, so other organizations may be best at doing "more of" that particular item. The same is true for the "less of" items. As such, it is not a critique of the current NASA program per se. Instead, it is a list that results from taking a step back and creating a summary from the author's perspective.

Many of these suggestions contain deliberate boldness for fomenting a healthy debate. The author offers the list in the spirit that room for improvement always exists. The ordering below for areas that need additional attention is in descending priority.

1. More of: Initial field evaluations in the next few years would be extremely valuable to judge the acceptability and performance of the already-developed automated conflict detection and resolution capabilities. Automated conflict detection and resolution is extremely complex, and it will need successive refinements. Refinements in simulation alone will be inadequate. Therefore, if it is true that relief is necessary for controllers by 2025 , researchers and developers cannot wait 10 years for the first field evaluations of these concepts. Presently, no agreed-to plans or schedule exists for even the first evaluation, understandably owing to the FAA's priorities in developing future versions of the En Route Automation Modernization System. However, without a focused and credible schedule on this principal and foundational element in NASA's research, the likelihood of having such an automated separation assurance in place at the end of the NextGen 20 -year horizon is low.

2. More of: To detect a satisfactory number of conflicts in time to resolve them safely and efficiently, today's flying airplanes will need to share more information than they do now with the ground automation. This is particularly true for climbing and descending aircraft. If weight, climb and descent profiles, and top-ofdescent were known on the ground, considerable progress could be made in detecting and resolving nearly $100 \%$ of potential conflicts. Decisions on what data, how often, and by what means need made.

3. Less of: There is a perception that many flight deck changes are required to increase capacity safely. Simulations indicate that significant increases in capacity are possible without such considerable and costly changes to the flight deck, such as ADS-B in, new flight management systems, and cockpit displays of traffic information. A ground-based trajectory prediction system with today's FANS-1/A datalink capability offers significant increases in capacity and efficiency. In the author's view, research should not stop on flightdeck improvements and solutions, as they may ultimately offer tractable and scalable solutions. However, the point is that these flightdeck improvements are likely not necessary, and much can be done with today's flightdeck, if the target capacity goal is $2-3 \mathrm{x}$.

4. More of: A reliable and safety-critical datalink is likely necessary for the full realization of automated separation assurance. The Mode S datalink developed previously by MIT Lincoln Laboratory, but not commissioned fully by the FAA due to the lack of identified need at the time, may offer such a capability. New secondary radar installations have Mode $S$ addressing capability, but they do not have the Mode $S$ datalink capability installed. If automated separation assurance is to become a future reality, additional attention needs paid to this foundational element. 
5. More of: The majority of the focus in the NASA program is on conceptually and efficiently increasing capacity to accommodate future increases in demand. Some efforts are underway to assess and consider the safety considerations and implications, and naturally, safety matters are in researchers and developers minds as they create and test new concepts. However, an uncomfortable amount of systematic safety attention exists in the present portfolio. Additional deliberation is essential on whether the portfolio needs rebalancing more towards investigating the safety questions.

6. More of: Significant improvements to traffic flow management today are possible by bringing computational tools to the operational floor. Solutions on deciding on the best national routes, assigning ground delay times to minimize overall delay, and using probabilistic techniques to allocate airplane release times in the likelihood that the weather will have cleared when reaching your destination, are essentially ready for use. However, fitting them seamlessly into existing plans, or living by the stipulation of not providing a traffic coordinator another separate display, are roadblocks that need overcome.

7. Less of: Portions of the existing research are, perhaps, 50 years out from realization. Typically, NASA's forte is applied research of a shorter term. If research addressing the mid-term stepping stones is satisfactory, then it may be appropriate for parts of the NASA portfolio to contain far-term, or 50-year-horizon research. However, if significant gaps exist for the stepping stones, then NASA should consider focusing on the shorter-term applied research. In the author's view, much of the surface optimization research falls in this category. Expecting precise and choreographed trajectories on the airport surface is likely feasible in the far term only. Instead, it may be possible to reorient the optimization to serve as a computational advisor to a human controller. The advisor would offer the next-best suggestion to reduce delay or increase capacity. This reorientation may better bridge the gap between today's and far-from-today's operations.

\section{Conclusions}

Ames has made many contributions to aid controllers in air traffic management. The Traffic Management Advisor is the most successful of these contributions with its nationwide deployment. Several other tools completed promising field tests, yet the FAA decided not to deploy them operationally. A few of these tools, such as Direct-To, are seeing a potential resurgence through other avenues.

Present air traffic management research at Ames covers a range of topics that include varying automation levels. All of these levels aim at reducing controller workload while increasing capacity, with an ultimate possible option of eliminating human responsibility for separation. Other automation can optimize traffic flows on the ground and in the air with the objective of increasing throughput without a concomitant increase in delay. Several technology transfers of existing research are underway and making a difference in the national airspace. These include enabling more continuous descent arrivals and improvements to tactical conflict detection.

For the future, early first-look evaluations in an operational context would offer considerable value to ongoing research, as simulations do not yet capture the significant complexities and interrelations present in the national airspace. For some of the present conflict detection and resolution research, considerable en route capacity benefits with today's flightdeck could accrue soon from early field tests. Redoubled efforts are essential to construct a convincing safety case for shifting the separation responsibility from a human to an automation system. Depending on the ultimate level of automation installed, part of that safety case may necessitate a highly reliable datalink to accommodate short-term conflict resolutions sent from ground automation.

Overall, NASA Ames continues to make many research contributions to improve air traffic management in the national airspace system. With steady financial support, increased stakeholder collaboration, skilled project management, and focused researcher attention on analysis, simulation, and operational testing, the research needs for the next-generation air-traffic-management system will be met.

\section{References}

1. Joint Planning and Development Office, "Next Generation Air Transportation System Integrated Plan," Dec. 2004.

2. Erzberger, H., Davis, T. J., and Green, S. M., "Design of Center-TRACON Automation System," AGARD Meeting on Machine Intelligence in Air Traffic Management, Berlin, Germany, 11-14 May 1993.

3. Denery, D. and Erzberger, H., "The Center-TRACON Automation System: Simulation and Field Testing," 
NASA TM 110366, Aug. 1995.

4. Erzberger, H., and Tobias, L., "A Time-Based Concept for Terminal-Area Traffic Management," Proceedings of the 1986 AGARD Conference No. 410 on Efficient Conduct of Individual Flights and Air Traffic, Brussels, Belgium, June 1986, pp. 52-1 - 52-14.

5. Slattery, R. A., "Terminal Area Trajectory Synthesis for Air Traffic Control Automation," American Control Conference, Seattle, WA, June 1995.

6. Nedell, W., Erzberger, H., and Neuman, F., "The Traffic Management Advisor," American Control Conference, San Diego, CA, 1990.

7. Harwood, K., and Sanford, B., "Denver TMA Assessment," NASA CR 4554, Oct. 1993.

8. Swenson, H.N., Hoang, T., Engelland, S., Vincent, D., Sanders, T., Sanford, B., and Heere, K., "Design and Operational Evaluation of the Traffic Management Advisor at the Fort Worth Air Route Traffic Control Center," $1^{\text {st }}$ USA/Europe Air Traffic Management R\&D Seminar, Saclay, France, June, 1997.

9. Wong, G.L., "The Dynamic Planner: The Sequencer, Scheduler, and Runway Allocator for Air Traffic Control Automation," NASA TM-2000-209586, Apr. 2000.

10. Federal Aviation Administration, "Free Flight Program Performance Metrics Results to Date: December 2003 Report," Dec. 2003.

11. Federal Aviation Administration, Traffic Mgmt Advisor-Single Cntr (TMA), Exhibit 300 FY2010, FAAXX603, 2009. http://www.dot.gov/exhibit300/2009/FAAXX603.pdf

12. Farley, T.C., Landry, S.J., Hoang, T., Nickelson, M., Levin, K.M., Rowe, D., and Welch, J.D., "Multi-Center Traffic Management Advisor: Operational Test Results," $5^{\text {th }}$ Aviation Technology, Integration \& Operations Conference, Arlington, VA, Sept. 2005.

13. Landry, S.J., Farley, T., Hoang, T., "Expanding the Use of Time-Based Metering: Multi-Center Traffic Management Advisor," $6^{\text {th }}$ USA/Europe Air Traffic Management Research and Development Seminar, Baltimore, MD, June 2005.

14. Green, S.M., and Vivona, R., "Field Evaluation of Descent Advisor Trajectory Prediction Accuracy," AIAA96-3764, AIAA Guidance, Navigation, and Control Conference, San Diego, CA, July 1996.

15. Green, S.M., Vivona, R.A., Grace, M.P., and Fang, T.C., "Field Evaluation of Descent Advisor Trajectory Prediction Accuracy for En-route Clearance Advisories," AIAA Guidance, Navigation, and Control Conference, Boston, MA, Aug. 1998.

16. Davis, T.J., Krzeczowski, K.J., and Bergh, C., "The Final Approach Spacing Tool," $13^{\text {th }}$ IFAC Symposium on Automatic Control in Aerospace, Palo Alto, CA, Sept. 1994.

17. Davis, T. J., Isaacson, D. R., Robinson III, J. E., den Braven, W., Lee, K. K., and Sanford, B., "Operational Field Test Results of the Passive Final Approach Spacing Tool," IFAC 8th Symposium on Transportation Systems, Chania, Greece, June 1997.

18. Erzberger, H., McNally, D., Foster, M., Chiu, D., and Stassart, P., "Direct-To Tool for En Route Controllers," ATM '99: IEEE Workshop on Advanced Technologies and Their Impact on Air Traffic Management in the $21^{\text {st }}$ Century, Capri, Italy, Nov. 1999.

19. McNally, D., Engelland, S., Bach, R., Chan, W., Brasil, C., Gong, C., Frey, J., and Vincent, D., "Operational Evaluation of the Direct-To Controller Tool," $4{ }^{\text {th }}$ USA/Europe Air Traffic Management R\&D Seminar, Santa Fe, NM, Dec. 2001.

20. Glass, B.J., "Automated Data Exchange and Fusion for Airport Surface Traffic Management," AIAA Guidance, Navigation, and Control Conference, New Orleans, LA, Aug. 1997.

21. Atkins, S., Jung, Y., Brinton, C., Stell, L., Carniol, T., and Rogowski, S., "Surface Management System Field Trial Results," AIAA 4 ${ }^{\text {th }}$ Aviation Technology, Integration and Operations Forum, Chicago, IL, Sept., 2004.

22. Atkins, S., Brinton, C., and Walton, D., "Functionalities, Displays, and Concept of Use for the Surface Management System," $21^{\text {st }}$ AIAA/IEEE Digital Avionics Systems Conference, Irvine, CA, Oct. 2002.

23. Bilimoria, K.D., Sridhar, B., Chatterji, G., Sheth, K.S., and Grabbe, S., "FACET: Future ATM Concepts Evaluation Tool," Air Traffic Control Quarterly, Vol. 9, No. 1, 2001, pp. 1-20.

24. Sridhar, B., Sheth, K., Smith, P., and Leber, W., "Migration of FACET from Simulation Environment to Dispatcher Decision Support System," Proceedings of the Digital Avionics Systems Conference, Washington, DC, Nov. 2005.

25. Sweet, D.N., Manikonda, V., Aronson, J.S., Roth, K., and Blake, M., "Fast-Time Simulation System for Analysis of Advanced Air Transportation Concepts," AIAA Modeling and Simulation Technologies Conference and Exhibit, Monterey, CA, Aug. 2002.

26. Meyn, L.A., Romer, T.F., Roth, K., Bjarke, L.J., and Hinton, S.E., "Preliminary Assessment of Future Operational Concepts Using the Airspace Concept Evaluation System," $4^{\text {th }}$ Aviation Technology, Integration 
and Operations Forum, Chicago, IL, Sept. 2004.

27. Zelinski, S. and Meyn, L., "Validating the Airspace Concept Evaluation System for Different Weather Days," AIAA Modeling and Simulation Technologies Conference and Exhibit, Keystone, CO, Aug. 2006.

28. Erzberger, H., "Automated Conflict Resolution for Air Traffic Control," $25^{\text {th }}$ International Congress of the Aeronautical Sciences, Hamburg, Germany, Sep. 2006.

29. Windhorst, R., Refai, M., and Karahan, S., "Convective Weather Avoidance with Uncertain Weather Forecasts," $28^{\text {th }}$ AIAA/IEEE Digital Avionics Systems Conference, Orlando, FL, Oct. 2009.

30. Erzberger, H., "The Automated Airspace Concept," 4th USA/Europe Air Traffic Management R\&D Seminar, Santa Fe, NM, USA, 3-7 Dec. 2001.

31. Farley, T.C., and Erzberger, H., "Fast-Time Simulation Evaluation of a Conflict Resolution Algorithm Under High Air Traffic Demand," 7th USA/Europe ATM 2007 R\&D Seminar, Barcelona, Spain, 2-5 July 2007.

32. Kupfer, M., Farley, T., Chu, Y., and Erzberger, H., "Automated Conflict Resolution - A Simulation-Based Sensitivity Study of Airspace and Demand," $26^{\text {th }}$ International Congress of the Aeronautical Sciences, Anchorage, AK, Sept. 2008.

33. Prevot, T., Homola, J., and Mercer, J., "Human-in-the-Loop Evaluation of Ground-Based Automated Separation Assurance for NextGen," $26^{\text {th }}$ International Congress of the Aeronautical Sciences, Anchorage, AK, Sept. 2008.

34. McNally, D., and Thipphavong, D., "Automated Separation Assurance in the Presence of Uncertainty," $26^{\text {th }}$ International Congress of the Aeronautical Sciences, Anchorage, AK, Sept. 2008.

35. Paielli, R.A., Erzberger, H., Chiu, D., Heere, K.R., "Tactical Conflict Alerting Aid for Air Traffic Controllers," Journal of Guidance, Control, and Dynamics, Vol., 32, No. 1, Jan-Feb, 2009.

36. Prevot, T., Homola, J., Mercer, J., Mainini, M., and Cabrall, C., "Initial Evaluation of NextGen Air/Ground Operations with Ground-Based Automated Separation Assurance," $8{ }^{\text {th }}$ USA/Europe Air Traffic Management Research and Development Seminar, Napa, CA, June 2009.

37. Coppenbarger, R.A., Mead, R.W., and Sweet, D.N., "Field Evaluation of the Tailored Arrivals Concept for Datalink-Enabled Continuous Descent Approach," $7^{\text {th }}$ AIAA Aviation Technology, Integration and Operations Conference, Belfast, Northern Ireland, Sept. 2007.

38. Chen, N.Y. and Sridhar, B., "Weather-Weighted Periodic Auto Regressive Models for Sector Demand Prediction," AIAA Guidance, Navigation, and Control Conference, Chicago, IL, Aug. 2009.

39. Chan, W. and Refai, M., "An Approach to Verify a Model for Translating Convective Weather Information to Air Traffic Management Impact," AIAA Aviation Technology, Integration, and Operations, Belfast, Northern Ireland, Sept. 2007.

40. Grabbe, S., Sridhar, B., and Mukherjee, A., "Integrated Traffic Flow Management Decision Making," AIAA Guidance, Navigation and Control Conference, Chicago, IL, Aug. 2009.

41. Mukherjee, A., Grabbe, S., and Sridhar, B., "Alleviating Airspace Restrictions through Strategic Control," AIAA Guidance, Navigation, and Control Conference, Honolulu, HI, Aug., 2008.

42. Mukherjee, A., Hansen, M., and Grabbe, S., "Ground Delay Program Planning Under Uncertainty in Airport Capacity," AIAA Guidance, Navigation, and Control Conference, Chicago, IL, Aug. 2009.

43. Grabbe, S. and Sridhar, B., "Congestion Management with an Aggregate Flow Model," AIAA Guidance, Navigation, and Control Conference, San Francisco, CA, Aug. 2005.

44. Sridhar, B., Grabbe, S., and Mukherjee, A., "Modeling and Optimization in Traffic Flow Management," Proceedings of the IEEE, Vol. 96, No. 12, Dec. 2008, pp. 1-29.

45. Rios, J. and Ross, K., "Massively Parallel Dantzig-Wolfe Decomposition Applied to Traffic Flow Scheduling," AIAA Guidance, Navigation and Control Conference, Chicago, IL, Aug. 2009.

46. Idris, H., Vivona, R., Penny, S., Krozel, J., and Bilimoria, K., "Operational Concept for Collaborative Traffic Flow Management based on Field Observations," AIAA $5^{\text {th }}$ Aviation, Technology, Integration, and Operations Conference, Arlington, VA, Sept. 2005.

47. Robinson, J., NASA Ames Research Center, Personal communication, 2009.

48. Verma, S., et. al., "Procedures for Off-Nominal Cases: Three Closely Spaced Parallel Runway Operations," $8^{\text {th }}$ USA/Europe Air Traffic Management Research and Development Seminar, Napa, CA, June 2009.

49. Kopardekar, P., Prevot, T., and Jastrzebski, M., "Traffic Complexity Measurement Under Higher Levels of Automation and Higher Traffic Densities," AIAA Guidance, Navigation, and Control Conference, Honolulu, HI, Aug. 2008.

50. Zelinski, S., "A Comparison of Algorithm Generated Sectorizations," $8^{\text {th }}$ USA/Europe Air Traffic Management Research and Development Seminar, Napa, CA, June, 2009.

51. Bloem, M., and Kopardekar, P., "Combining Airspace Sectors for the Efficient Use of Air Traffic Control 
Resources," AIAA Guidance, Navigation, and Control Conference, Honolulu, HI, Aug. 2008.

52. Rathinam, S., Montoya, J., and Jung, Y., "An Optimization Model for Reducing Aircraft Taxi Times at the Dallas Fort Worth International Airport," $26^{\text {th }}$ International Congress of the Aeronautical Sciences, Anchorage, AK, Sept. 2008.

53. Rathinam, S., Wood, Z., Sridhar, B., and Jung, Y., "A Generalized Dynamic Programming Approach for a Departure Scheduling Problem," AIAA Guidance, Navigation, and Control Conference, Chicago, IL, Aug. 2009.

54. Gupta, G., Malik, W., and Jung, Y., “A Mixed Integer Linear Program for Airport Departure Scheduling,” $9^{\text {th }}$ AIAA Aviation Technology, Integration, and Operations Conference, Hilton Head, SC, Sept. 2009.

55. Verma, S., Kozon, T., Cheng, V., and Ballinger, D., "Changes in Roles/Responsibilities of Air Traffic Control Under Precision Taxiing," $26^{\text {th }}$ AIAA/IEEE Digital Avionics Systems Conference, Dallas, TX, Oct. 2007.

56. Vivona, R.A., Cate, K.T., and Green, S.G., “Abstraction Techniques for Capturing and Comparing Trajectory Predictor Capabilities and Requirements," AIAA Guidance, Navigation and Control Conference, Honolulu, HI, Aug. 2008.

57. Federal Aviation Administration, “Aviation Forecasts,” http://www.faa.gov/data_research/aviation/, 2009 